\title{
The Relevance of Measure and Probability, and Definition of Completeness of Probability
}

\author{
Bo Zhang \\ Shinshu University \\ Nagano, Japan
}

\author{
Hiroshi Yamazaki \\ Shinshu University \\ Nagano, Japan
}

\author{
Yatsuka Nakamura \\ Shinshu University \\ Nagano, Japan
}

Summary. In this article, we first discuss the relation between measure defined using extended real numbers and probability defined using real numbers. Further, we define completeness of probability, and its completion method, and also show that they coincide with those of measure.

MML identifier: PROB_4, version: 7.6.01 4.53.937

The articles [18], [20], [2], [3], [5], [1], [12], [15], [21], [8], [19], [17], [4], [9], [14], [23], [6], [11], [16], [22], [10], [7], and [13] provide the notation and terminology for this paper.

For simplicity, we adopt the following convention: $n$ denotes a natural number, $X$ denotes a set, $A_{1}$ denotes a sequence of subsets of $X, S_{1}$ denotes a $\sigma$-field of subsets of $X, X_{1}$ denotes a sequence of subsets of $S_{1}, O_{1}$ denotes a non empty set, $S_{2}$ denotes a $\sigma$-field of subsets of $O_{1}, A_{2}$ denotes a sequence of subsets of $S_{2}$, and $P$ denotes a probability on $S_{2}$.

Let us consider $X, S_{1}, X_{1}, n$. Then $X_{1}(n)$ is an element of $S_{1}$.

Next we state two propositions:

(1) $\operatorname{rng} X_{1} \subseteq S_{1}$.

(2) For every function $f$ holds $f$ is a sequence of subsets of $S_{1}$ iff $f$ is a function from $\mathbb{N}$ into $S_{1}$.

The scheme LambdaSigmaSSeq deals with a set $\mathcal{A}$, a $\sigma$-field $\mathcal{B}$ of subsets of $\mathcal{A}$, and a unary functor $\mathcal{F}$ yielding an element of $\mathcal{B}$, and states that:

There exists a sequence $f$ of subsets of $\mathcal{B}$ such that for every $n$ holds $f(n)=\mathcal{F}(n)$ 
for all values of the parameters.

Let us consider $X$. Note that there exists a sequence of subsets of $X$ which is disjoint valued.

Let us consider $X, S_{1}$. Note that there exists a sequence of subsets of $S_{1}$ which is disjoint valued.

One can prove the following propositions:

(3) For all subsets $A, B$ of $X$ there exists $A_{1}$ such that $A_{1}(0)=A$ and $A_{1}(1)=B$ and for every $n$ such that $n>1$ holds $A_{1}(n)=\emptyset$.

(4) Let $A, B$ be subsets of $X$. Suppose $A$ misses $B$ and $A_{1}(0)=A$ and $A_{1}(1)=B$ and for every $n$ such that $n>1$ holds $A_{1}(n)=\emptyset$. Then $A_{1}$ is disjoint valued and $\bigcup A_{1}=A \cup B$.

(5) Let $S$ be a non empty set. Then $S$ is a $\sigma$-field of subsets of $X$ if and only if the following conditions are satisfied:

(i) $S \subseteq 2^{X}$,

(ii) for every sequence $A_{1}$ of subsets of $X$ such that for every $n$ holds $A_{1}(n) \in S$ holds $\bigcup A_{1} \in S$, and

(iii) for every subset $A$ of $X$ such that $A \in S$ holds $A^{\mathrm{c}} \in S$.

(6) For all events $A, B$ of $S_{2}$ holds $P(A \backslash B)=P(A \cup B)-P(B)$.

(7) For all events $A, B$ of $S_{2}$ such that $A \subseteq B$ and $P(B)=0$ holds $P(A)=0$.

(8) For every $n$ holds $P\left(A_{2}(n)\right)=0$ iff $P\left(\bigcup A_{2}\right)=0$.

(9) For every set $A$ such that $A \in \operatorname{rng} A_{2}$ holds $P(A)=0$ iff $P\left(\bigcup \operatorname{rng} A_{2}\right)=0$.

(10) For every function $s_{1}$ from $\mathbb{N}$ into $\mathbb{R}$ and for every function $E_{1}$ from $\mathbb{N}$ into $\overline{\mathbb{R}}$ such that $s_{1}=E_{1}$ holds $\left(\sum_{\alpha=0}^{\kappa}\left(s_{1}\right)(\alpha)\right)_{\kappa \in \mathbb{N}}=\operatorname{Ser} E_{1}$.

(11) Let $s_{1}$ be a function from $\mathbb{N}$ into $\mathbb{R}$ and $E_{1}$ be a function from $\mathbb{N}$ into $\overline{\mathbb{R}}$. If $s_{1}=E_{1}$ and $s_{1}$ is upper bounded, then $\sup s_{1}=\sup \operatorname{rng} E_{1}$.

(12) Let $s_{1}$ be a function from $\mathbb{N}$ into $\mathbb{R}$ and $E_{1}$ be a function from $\mathbb{N}$ into $\overline{\mathbb{R}}$. If $s_{1}=E_{1}$ and $s_{1}$ is lower bounded, then $\inf s_{1}=\inf \operatorname{rng} E_{1}$.

(13) Let $s_{1}$ be a function from $\mathbb{N}$ into $\mathbb{R}$ and $E_{1}$ be a function from $\mathbb{N}$ into $\overline{\mathbb{R}}$. If $s_{1}=E_{1}$ and $s_{1}$ is non-negative and summable, then $\sum s_{1}=\sum E_{1}$.

(14) $P$ is a $\sigma$-measure on $S_{2}$.

Let us consider $O_{1}, S_{2}, P$. The functor P2M $P$ yields a $\sigma$-measure on $S_{2}$ and is defined as follows:

(Def. 1) $\quad \mathrm{P} 2 \mathrm{M} P=P$.

One can prove the following proposition

(15) Let $X$ be a non empty set, $S$ be a $\sigma$-field of subsets of $X$, and $M$ be a $\sigma$-measure on $S$. If $M(X)=\overline{\mathbb{R}}(1)$, then $M$ is a probability on $S$.

Let $X$ be a non empty set, let $S$ be a $\sigma$-field of subsets of $X$, and let $M$ be a $\sigma$-measure on $S$. Let us assume that $M(X)=\overline{\mathbb{R}}(1)$. The functor M2P $M$ yielding a probability on $S$ is defined as follows: 
(Def. 2) $\quad \mathrm{M} 2 \mathrm{P} M=M$.

One can prove the following propositions:

(16) If $A_{1}$ is non-decreasing, then the partial unions of $A_{1}=A_{1}$.

(17) Suppose $A_{1}$ is non-decreasing. Then (the partial diff-unions of $\left.A_{1}\right)(0)=$ $A_{1}(0)$ and for every $n$ holds (the partial diff-unions of $\left.A_{1}\right)(n+1)=A_{1}(n+$ 1) $\backslash A_{1}(n)$.

(18) If $A_{1}$ is non-decreasing, then for every $n$ holds $A_{1}(n+1)=$ (the partial diff-unions of $\left.A_{1}\right)(n+1) \cup A_{1}(n)$.

(19) If $A_{1}$ is non-decreasing, then for every $n$ holds (the partial diff-unions of $\left.A_{1}\right)(n+1)$ misses $A_{1}(n)$.

(20) If $X_{1}$ is non-decreasing, then the partial unions of $X_{1}=X_{1}$.

(21) Suppose $X_{1}$ is non-decreasing. Then (the partial diff-unions of $\left.X_{1}\right)(0)=$ $X_{1}(0)$ and for every $n$ holds (the partial diff-unions of $\left.X_{1}\right)(n+1)=X_{1}(n+$ 1) $\backslash X_{1}(n)$.

(22) If $X_{1}$ is non-decreasing, then for every $n$ holds (the partial diff-unions of $\left.X_{1}\right)(n+1)$ misses $X_{1}(n)$.

Let us consider $O_{1}, S_{2}, P$. We say that $P$ is complete on $S_{2}$ if and only if:

(Def. 3) For every subset $A$ of $O_{1}$ and for every set $B$ such that $B \in S_{2}$ holds if $A \subseteq B$ and $P(B)=0$, then $A \in S_{2}$.

Next we state the proposition

(23) $P$ is complete on $S_{2}$ iff P2M $P$ is complete on $S_{2}$.

Let us consider $O_{1}, S_{2}, P$. A subset of $O_{1}$ is called a set with measure zero w.r.t. $P$ if:

(Def. 4) There exists a set $A$ such that $A \in S_{2}$ and it $\subseteq A$ and $P(A)=0$.

We now state three propositions:

(24) Let $Y$ be a subset of $O_{1}$. Then $Y$ is a set with measure zero w.r.t. $P$ if and only if $Y$ is a set with measure zero w.r.t. P2M $P$.

(25) $\emptyset$ is a set with measure zero w.r.t. $P$.

(26) Let $B_{1}, B_{2}$ be sets. Suppose $B_{1} \in S_{2}$ and $B_{2} \in S_{2}$. Let $C_{1}, C_{2}$ be sets with measure zero w.r.t. $P$. If $B_{1} \cup C_{1}=B_{2} \cup C_{2}$, then $P\left(B_{1}\right)=P\left(B_{2}\right)$.

Let us consider $O_{1}, S_{2}, P$. The functor $\operatorname{COM}\left(S_{2}, P\right)$ yields a non empty family of subsets of $O_{1}$ and is defined by the condition (Def. 5).

(Def. 5) Let $A$ be a set. Then $A \in \operatorname{COM}\left(S_{2}, P\right)$ if and only if there exists a set $B$ such that $B \in S_{2}$ and there exists a set $C$ with measure zero w.r.t. $P$ such that $A=B \cup C$.

Next we state two propositions:

(27) For every set $C$ with measure zero w.r.t. $P$ holds $C \in \operatorname{COM}\left(S_{2}, P\right)$.

(28) $\operatorname{COM}\left(S_{2}, P\right)=\operatorname{COM}\left(S_{2}, \mathrm{P} 2 \mathrm{M} P\right)$. 
Let us consider $O_{1}, S_{2}, P$ and let $A$ be an element of $\operatorname{COM}\left(S_{2}, P\right)$. The functor $\mathrm{P}_{\mathrm{COM}} 2 \mathrm{M}_{\mathrm{COM}} A$ yields an element of $\operatorname{COM}\left(S_{2}, \mathrm{P} 2 \mathrm{M} P\right)$ and is defined by:

(Def. 6) $\quad \mathrm{P}_{\mathrm{COM}} 2 \mathrm{M}_{\mathrm{COM}} A=A$.

Next we state the proposition

(29) $S_{2} \subseteq \operatorname{COM}\left(S_{2}, P\right)$.

Let us consider $O_{1}, S_{2}, P$ and let $A$ be an element of $\operatorname{COM}\left(S_{2}, P\right)$. The functor ProbPart $A$ yielding a non empty family of subsets of $O_{1}$ is defined by:

(Def. 7) For every set $B$ holds $B \in \operatorname{ProbPart} A$ iff $B \in S_{2}$ and $B \subseteq A$ and $A \backslash B$ is a set with measure zero w.r.t. $P$.

We now state several propositions:

(30) For every element $A$ of $\operatorname{COM}\left(S_{2}, P\right)$ holds ProbPart $A=$ MeasPart $\mathrm{P}_{\mathrm{COM}} 2 \mathrm{M}_{\mathrm{COM}} A$.

(31) For every element $A$ of $\operatorname{COM}\left(S_{2}, P\right)$ and for all sets $A_{1}, A_{3}$ such that $A_{1} \in \operatorname{ProbPart} A$ and $A_{3} \in \operatorname{ProbPart} A$ holds $P\left(A_{1}\right)=P\left(A_{3}\right)$.

(32) For every function $F$ from $\mathbb{N}$ into $\operatorname{COM}\left(S_{2}, P\right)$ there exists a sequence $B_{3}$ of subsets of $S_{2}$ such that for every $n$ holds $B_{3}(n) \in \operatorname{ProbPart} F(n)$.

(33) Let $F$ be a function from $\mathbb{N}$ into $\operatorname{COM}\left(S_{2}, P\right)$ and $B_{3}$ be a sequence of subsets of $S_{2}$. Then there exists a sequence $C_{3}$ of subsets of $O_{1}$ such that for every $n$ holds $C_{3}(n)=F(n) \backslash B_{3}(n)$.

(34) Let $B_{3}$ be a sequence of subsets of $O_{1}$. Suppose that for every $n$ holds $B_{3}(n)$ is a set with measure zero w.r.t. $P$. Then there exists a sequence $C_{3}$ of subsets of $S_{2}$ such that for every $n$ holds $B_{3}(n) \subseteq C_{3}(n)$ and $P\left(C_{3}(n)\right)=0$.

(35) Let $D$ be a non empty family of subsets of $O_{1}$. Suppose that for every set $A$ holds $A \in D$ iff there exists a set $B$ such that $B \in S_{2}$ and there exists a set $C$ with measure zero w.r.t. $P$ such that $A=B \cup C$. Then $D$ is a $\sigma$-field of subsets of $O_{1}$.

Let us consider $O_{1}, S_{2}, P$. Then $\operatorname{COM}\left(S_{2}, P\right)$ is a $\sigma$-field of subsets of $O_{1}$.

Let us consider $O_{1}, S_{2}, P$. We see that the set with measure zero w.r.t. $P$ is an event of $\operatorname{COM}\left(S_{2}, P\right)$.

Next we state two propositions:

(36) For every set $A$ holds $A \in \operatorname{COM}\left(S_{2}, P\right)$ iff there exist sets $A_{1}, A_{3}$ such that $A_{1} \in S_{2}$ and $A_{3} \in S_{2}$ and $A_{1} \subseteq A$ and $A \subseteq A_{3}$ and $P\left(A_{3} \backslash A_{1}\right)=0$.

(37) Let $C$ be a non empty family of subsets of $O_{1}$. Suppose that for every set $A$ holds $A \in C$ iff there exist sets $A_{1}, A_{3}$ such that $A_{1} \in S_{2}$ and $A_{3} \in S_{2}$ and $A_{1} \subseteq A$ and $A \subseteq A_{3}$ and $P\left(A_{3} \backslash A_{1}\right)=0$. Then $C=\operatorname{COM}\left(S_{2}, P\right)$.

Let us consider $O_{1}, S_{2}, P$. The functor $\operatorname{COM}(P)$ yields a probability on $\operatorname{COM}\left(S_{2}, P\right)$ and is defined as follows: 
(Def. 8) For every set $B$ such that $B \in S_{2}$ and for every set $C$ with measure zero w.r.t. $P$ holds $(\operatorname{COM}(P))(B \cup C)=P(B)$.

One can prove the following propositions:

(38) $\operatorname{COM}(P)=\mathrm{COM}(\mathrm{P} 2 \mathrm{M} P)$.

(39) $\operatorname{COM}(P)$ is complete on $\operatorname{COM}\left(S_{2}, P\right)$.

(40) For every event $A$ of $S_{2}$ holds $P(A)=(\operatorname{COM}(P))(A)$.

(41) For every set $C$ with measure zero w.r.t. $P$ holds $(\operatorname{COM}(P))(C)=0$.

(42) For every element $A$ of $\operatorname{COM}\left(S_{2}, P\right)$ and for every set $B$ such that $B \in$ ProbPart $A$ holds $P(B)=(\operatorname{COM}(P))(A)$.

\section{REFERENCES}

[1] Grzegorz Bancerek. The fundamental properties of natural numbers. Formalized Mathematics, 1(1):41-46, 1990.

[2] Grzegorz Bancerek. König's theorem. Formalized Mathematics, 1(3):589-593, 1990.

[3] Józef Białas. Completeness of the $\sigma$-additive measure. Measure theory. Formalized Mathematics, 2(5):689-693, 1991.

[4] Józef Białas. Infimum and supremum of the set of real numbers. Measure theory. Formalized Mathematics, 2(1):163-171, 1991.

[5] Józef Białas. Series of positive real numbers. Measure theory. Formalized Mathematics, 2(1):173-183, 1991.

[6] Józef Białas. The $\sigma$-additive measure theory. Formalized Mathematics, 2(2):263-270, 1991.

[7] Józef Białas. Some properties of the intervals. Formalized Mathematics, 5(1):21-26, 1996.

[8] Czesław Byliński. Functions and their basic properties. Formalized Mathematics, 1(1):5565, 1990.

[9] Czesław Byliński. Functions from a set to a set. Formalized Mathematics, 1(1):153-164, 1990.

[10] Czesław Byliński. Some basic properties of sets. Formalized Mathematics, 1(1):47-53, 1990.

[11] Czesław Byliński and Piotr Rudnicki. Bounding boxes for compact sets in $\mathcal{E}^{2}$. Formalized Mathematics, 6(3):427-440, 1997.

[12] Jarosław Kotowicz. Convergent sequences and the limit of sequences. Formalized Mathematics, 1(2):273-275, 1990.

[13] Andrzej Nȩdzusiak. Probability. Formalized Mathematics, 1(4):745-749, 1990.

[14] Andrzej Nędzusiak. $\sigma$-fields and probability. Formalized Mathematics, 1(2):401-407, 1990.

[15] Beata Padlewska. Families of sets. Formalized Mathematics, 1(1):147-152, 1990.

[16] Konrad Raczkowski and Andrzej Nȩdzusiak. Series. Formalized Mathematics, 2(4):449452, 1991

[17] Andrzej Trybulec. Subsets of complex numbers. To appear in Formalized Mathematics.

[18] Andrzej Trybulec. Tarski Grothendieck set theory. Formalized Mathematics, 1(1):9-11, 1990.

[19] Zinaida Trybulec. Properties of subsets. Formalized Mathematics, 1(1):67-71, 1990.

[20] Edmund Woronowicz. Relations and their basic properties. Formalized Mathematics, 1(1):73-83, 1990.

[21] Edmund Woronowicz. Relations defined on sets. Formalized Mathematics, 1(1):181-186, 1990

[22] Bo Zhang, Hiroshi Yamazaki, and Yatsuka Nakamura. Inferior limit and superior limit of sequences of real numbers. Formalized Mathematics, 13(3):375-381, 2005.

[23] Bo Zhang, Hiroshi Yamazaki, and Yatsuka Nakamura. Set sequences and monotone class. Formalized Mathematics, 13(4):435-441, 2005.

Received November 23, 2005 